\title{
Innovative Design and Manufacture of "S" Type Carbon-Free Cars
}

\author{
Jianwei Liu ${ }^{1}$, Yuhan $\mathrm{Li}^{2}$ and Nianjiong Yang ${ }^{3}$ \\ ${ }^{1}$ Engineering Training Center of Guilin University of Electronic Technology, Guilin 541004, China \\ ${ }^{2}$ Electromechanical Engineering College of Guilin University of Electronic Technology, Guilin 541004, China \\ ${ }^{3}$ Guangxi University of Science and Technology, Guangxi key laboratory of Automobile Components and Vehicle technology, 545006 \\ Liuzhou, China
}

\begin{abstract}
Based on the new rules of the 4th national college students' engineering and comprehensive training ability competition, established three-dimensional model using software UG NX, designed a kind of "S" type carbon-free, it can adapt to various poles' distance and easy assembling and debugging.Focus on variable pitch mechanism and steering mechanism's designing, and by motion simulation verify its rationality, the simulation analysis showed the car trajectory accords with a requirement. Finally,processed parts,assembled and debugged of the cars.Practice has proved that the design of the car conform to the requirements of the game, is reasonable, assembling outfit is convenient, easy to debug, can meet the requirements of a variety of stem from, smooth finish and get good grades.
\end{abstract}

\section{Introduction}

Carbon-free car, a kind of three-wheel cars, is driven by given gravitational potential energy, which has the function of continuous obstacle avoidance. The "S"shaped circuit requires the car to be able to automatically bypass the obstacles on the track when the car is moving. Obstacle for the diameter of $20 \mathrm{~mm}$, high $200 \mathrm{~mm}$ round bar, along the track line and other distance placed. It is different from the previous game rules that the obstacle space to expand the scope of $700 \mathrm{~mm} \sim 1300 \mathrm{~mm}$ and require to the car on-site dismantling debugging before the game. In the past, the carbon free cars [1]-[4] are only one kind of transmission ratio, can not meet the current requirements of the game. Lu Jian [5] designed a carbonfree car which can adjust the transmission ratio, although it can adapt to the "S"-shaped path of different rod distance, but it is difficult to change the transmission ratio due to the complexity of the structure. Therefore, it is urgent to design a kind of variable distance mechanism which is convenient to adjust many kinds of transmission ratio, and it is convenient to disassemble and adjust the steering mechanism.

\section{Integrated design of the carbon-free car}

According to the rules of the three wheeled structure and to achieve the " $\mathrm{S}$ " shape of the line shape, the overall design of the car as shown in Fig. 1, including the frame, the transmission part, the steering part, the fine-tuning part of the 4 parts.

The principle is that lifting the weight to a specified height, then weight falls automatically due to the gravity.The flexible cord in the weight will change direction of tension by the installation of pulley in the pulley seat, and the other end of the rope will wind on the driving mechanism. The movement is transferred to the rear wheel shaft, which drives the rear wheel to rotate, thereby completing the movement of the trolley.The front wheel rotates to the left or to the right according to the sine period to rotate through the steering mechanism, which causes the car to walk out of the S shape path and avoid the obstacle rod which is arranged at a certain distance.

In addition to the standard thread parts and bearings of standard parts, the rest of the car are required students to manufacture their own, so must first consider the car material. According to the previous experience in design and processing [6], select aluminum body frame.

\section{Design of transmission part}

As shown in Fig. 1, the transmission part by pulley, wire wheel, wheel, gear and small gear adjustment and back axle, etc. Flexible rope through the pulley lead the line wheel to rotate, and the coaxial with the big gear to rotate together, so as to drive the meshed with the adjusting gear, rotate the pinion gear. The pinion drives the rear axle to rotate. So that the mounting in the rear ends of the two rear wheels rotate, the car forward. 


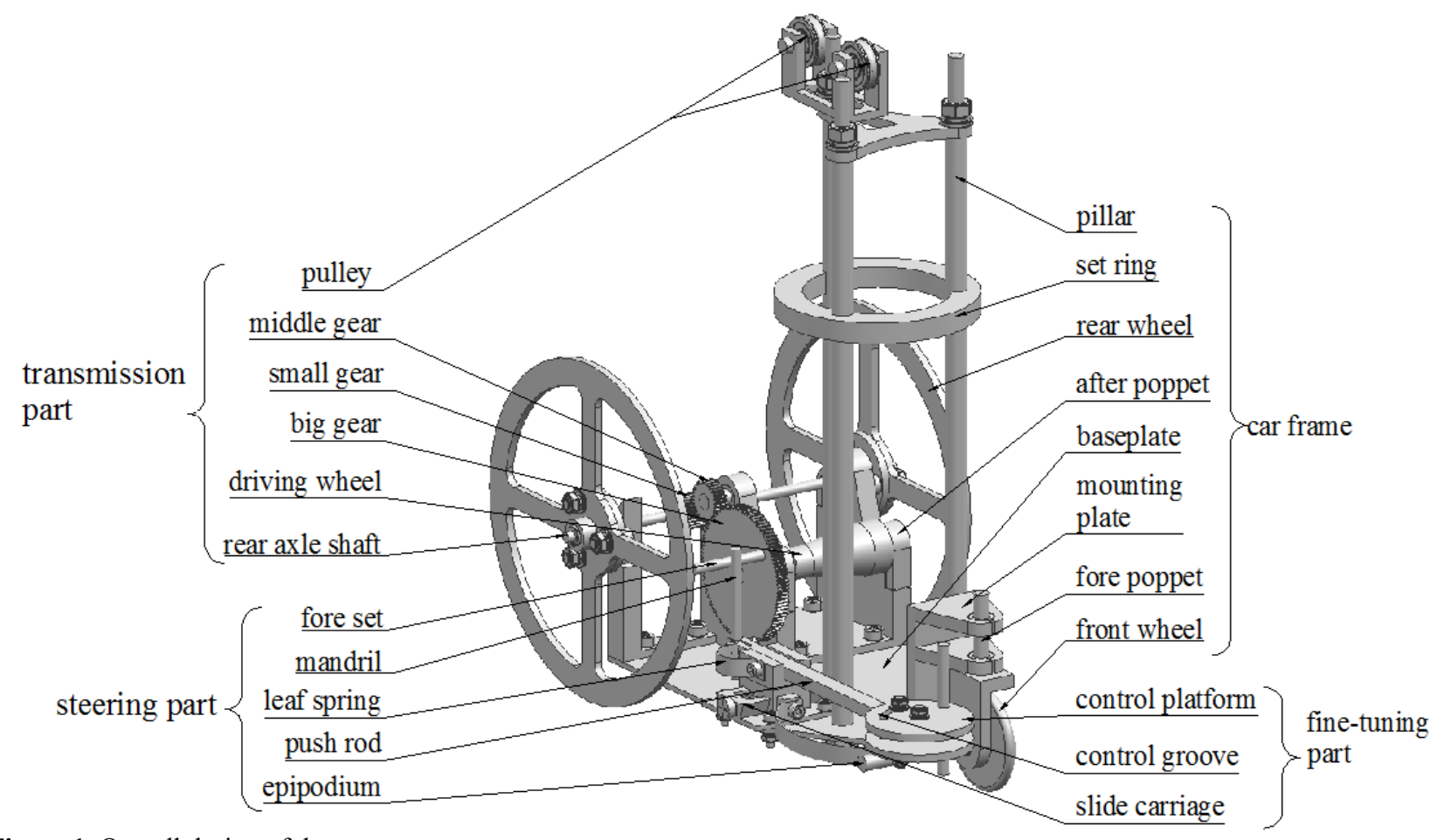

Figure 1. Overall design of the car

\subsection{Calculation of transmission ratio}

The game provides a random sample of obstacles in the range of $700 \mathrm{~mm} \sim 1300 \mathrm{~mm}$. According to the debugging experience, the car can meet the biggest obstacle distance of $200 \mathrm{~mm}$ by adjusting its own steering angle. So the range is divided into three groups: $700 \mathrm{~mm} \sim 900 \mathrm{~mm}$, $900 \mathrm{~mm} \sim 1100 \mathrm{~mm}, 1100 \mathrm{~mm} \sim 1300 \mathrm{~mm}$, the median values of each group were $800 \mathrm{~mm}, 1000 \mathrm{~mm}$ and $1200 \mathrm{~mm}$, respectively, and The optimal amplitude of the "S"-shape trajectory is $350 \mathrm{~mm}$. Using CAD and other drawing software for spline curve fitting trolley walking route, as shown in Fig. 2.

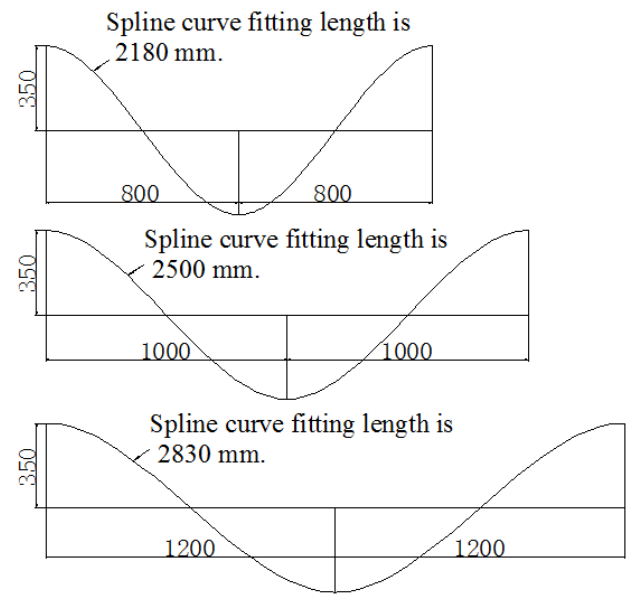

Figure 2. Movement track diagram of the car

Measuring the length of the spline curve can be obtained from the three space under the car a cycle of the theoretical distance respectively $2180 \mathrm{~mm}, 2500 \mathrm{~mm}$, $2830 \mathrm{~mm}$.
The relationship between the transmission ratio and the distance of a cycle:

$$
S=i \times \pi D
$$

where $S$ is the distance, $2180 \mathrm{~mm}, 2500 \mathrm{~mm}$ and $2830 \mathrm{~mm}$, respectively; $i$ is the transmission ratio; $D$ is the rear wheel diameter.

By formula (1) can calculate transmission ratio $i_{1}=5.3$, $i_{2}=6.1, i_{3}=6.9$, when the distance between $800 \mathrm{~mm}$, $1000 \mathrm{~mm}, 1200 \mathrm{~mm}$, respectively. The number of gear teeth is 120 , and the number of small gear teeth is 23,20 and 17.

\subsection{Design of variable pitch mechanism}

In order to adapt to the different distance, the design of the car with a variable pitch mechanism, as shown in Fig. 3. It only need to replace a small gear, and adjust the position of the gear to achieve the three gears meshing. Compared with the multi group gear meshing method [2], the operation is simple, no need to adjust the position of the gear support to make the gear mesh.

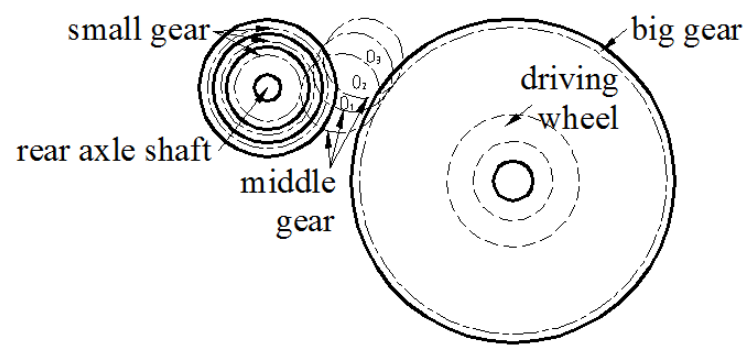

Figure 3. Principle diagram of the variable pitch institutions

\section{Design of steering part}


The "S"-shaped carbon free car commonly used in the steering mechanism has cam mechanism [7], incomplete gear mechanism, crank rocker mechanism, etc. This car using the sine mechanism, as shown in Fig. 1, steering part is composed of the top of the column, a push rod, a push rod, a spring piece and a front axle. The kinematic diagram [8] of the steering part as shown in Fig. 4.

When the big gear rotates, eccentric top column which fixed on the big gear followed to make circular motion. The front and back straight line motion of the push rod is driven by the eccentric top post, which drives the push rod to slide. The push rod is arranged in the slider and the two ends are respectively connected with the push rod and the front axle is in contact with $C$ and $D$. The top rod transfer to the front, so that the front axle to point $\mathrm{e}$ for the center for small angle swing back and forth, so as to realize the front left and right steering.

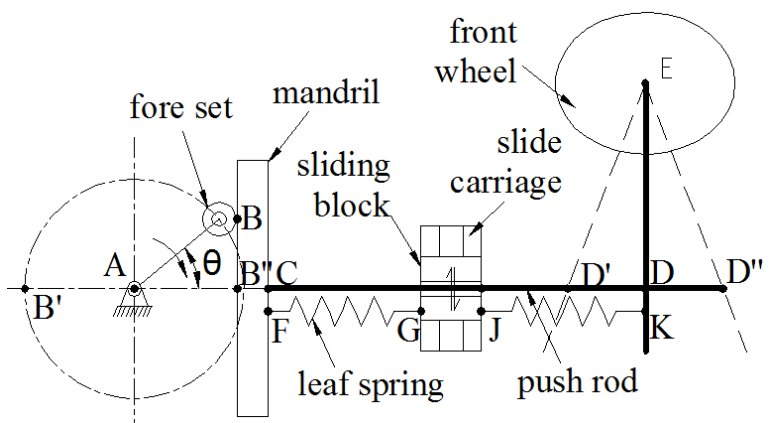

Figure 4. Steering mechanism kinematic sketch

As shown in Fig. 4, When the rocker $A B$ moves to $B^{\prime}$ and $B^{\prime \prime}$ position, the push rod has just arrived at the closest point $D^{\prime}$ and the farthest point $D^{\prime \prime}$. Assume that the length of the crank $A B$ is $l_{1}$, the $D^{\prime} D^{\prime \prime}$ is $2 l_{1}$. Assuming the length $D E$ is $l_{2}$, and the slider is in the middle position of the sliding plate, the front wheel rotation angle as

$$
\angle D^{\prime} E D^{\prime \prime}=2 \arctan \frac{l_{1}}{l_{2}}
$$

The maximum distance that the slider can be adjusted up and down is $l_{3}$, then the maximum $\psi$ and minimum values $\varphi$ for the front wheel angle as:

$$
\begin{gathered}
\psi=\angle D^{\prime} E D_{\text {max }}^{\prime \prime}=2 \arctan \frac{l_{1}-l_{3}}{l_{2}} \\
\varphi=\angle D^{\prime} E D_{\text {min }}^{\prime \prime}=2 \arctan \frac{l_{1}+l_{3}}{l_{2}}
\end{gathered}
$$

Different from the previous design of the steering mechanism, the car designed the compression spring [8] on the basis of the sine mechanism. Due to the effects of the front axle, front push rod spring and the front wheel shaft to maintain contact, and the rear ejector rod is in contact with an eccentric top post because of the action of the push rod spring. So it can keep gapless between the push rod with the front axle, also can maintain gapless between the push rod and the top pillar, making a smooth shift, and keeps the advantages of the sine mechanism. In addition, in order to overcome the uneven force of the spring plate, the two material and the same size spring piece are selected.

In ensuring the accuracy of processing and assembly accuracy, the car can be driven by the standard " $\mathrm{S}$ "shaped track, and do not need to adjust to find a balance position.

\section{Design of fine tuning part}

In order to make the car to avoid obstacles more accurate, fine adjustment mechanism [9] is needed to adjust the front wheel swing angle range. As shown in Fig. 5, tuning part is composed of sliding board, sliding block, regulating tank, adjusting plate and the like. The sliding block is arranged on the sliding plate. Using screw rod device as device for regulating horizontal movement of slide plate. The trimming principle is by turning screwsteering mechanism of lateral movement, change the top bar and the position of the front wheel axis to change the front wheel angular size, adjusting the amplitude of the trolley routes, and achieve precise fine-tuning of trolley routes. Meanwhile, in regulating trimmer slot is opened on, putting the front-end and adjusting connection point is located in the fine tuning of the disc slot.

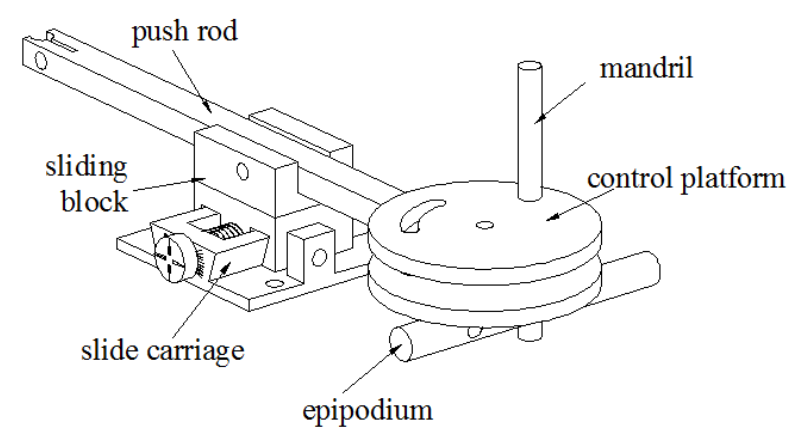

Figure 5. Fine adjustment mechanism

\section{Conclusions}

The overall innovative design for Carbon-free car is achieved, and the analysis of its structure and movement aimed at the parts of transmission, steering and fine tune is also completed in this study. For the pitch mechanism, it is equipped with many advantages, such as the transmission ratio adjusted easily, handling simply and disassembling and debugging conveniently. The precise control of track route is realized by the micro-adjusting mechanism providing two different measures, i.e. the horizontal adjusting mechanism and fine-tuning slots.

The practice proved that the car has the characteristics of simple structure, adjusting easily and the adaptation for multi-spacing, and provide a certain reference for the design of Carbon-free car under the new rules.

\section{Acknowledgment}

The authors are thankful to the Guangxi Natural Science Foundation (Grant no: 2013GXNSFBA019245), Science and technology research project of Guangxi University (Grant no: KY2015YB095), the Opening Project of 
Guangxi Key Laboratory of Automobile Components and Vehicle Technology, Guangxi University of Science and Technology (No.2014KFMS05), and the graduate student scientific research innovation projects of guilin university of electronic technology(Grant no: YJCXS201504)

\section{References}

1. Ji Yuan-jin, Ren Li-hui. Journal of Machine Design. Innovationa lstructure designo fcarbon-freecar byusing center-changed geart echnique. 14, 9 (2013)

2. Zou Guang-ming, Yang Xiu-guang. Research on the potential energy-driven car with energy stored by vortex coil spring. 7, 32-35, (2012)

3. Wang Bin, Wang Yan, Li Run-lian.Innovational design of carbon-free car. 2, 59-62, (2012).

4. Zhou Cheng, Gao Kun-shan, Ju Chen-ming, Zhang Ya-li. Dynamic Simulation of Carbon-Free Caron ADAMS. 8, 197-200, (2015).
5. Lu Jian, Wei De-qiang, Sui Xin-meng. An S-shaped path walking shift variable pitch carbon-free car:China, 201410839319.3[P]. (2015)

6. Chen Xiao-dong, Shi Yan-nan, Zhang Li-li. The Design, Manufacture and Innovative Practice of Carbon-free Car. 2, 92-295, (2013).

7. Zhang Chun, Zheng Ying-bin, Ma Yong-chang. Wide-angle turning performance optimization of gravitational potential energy driven three-wheeled car. 10, 50-55, (2014).

8. Liu Run, Zhu Xian -yong, Li Zhi -dong.Carbon -free car steering mechanism modeling and simulation based on spring constraint. 9, 24-27, (2013).

9. Li Yu-han, Liu Jian-wei, Ye Zi-lon. A kind of can adapt to different stem from carbon-free car Sshaped path:China, 201510175306.5[P], (2015) 